\title{
A novel laser guide star: Projected Pupil Plane Pattern
}

\author{
Huizhe Yang ${ }^{\mathrm{a}}$, Nazim Barmal ${ }^{\mathrm{a}}$, Richard Myers ${ }^{\mathrm{a}}$, David F. Buscher ${ }^{\mathrm{b}}$, Aglae Kellerer ${ }^{\mathrm{c}}$, Tim \\ Morris $^{\mathrm{a}}$, and Alastair Basden ${ }^{\mathrm{a}}$ \\ ${ }^{a}$ Department of Physics, Centre for Advanced Instrumentation, University of Durham, South \\ Road, Durham DH1 3LE, UK \\ ${ }^{\mathrm{b}}$ University of Cambridge, Department of Physics, Cavendish Laboratories, Cambridge CB3 \\ OHE, UK \\ ${ }^{c}$ ESO, Garching bei München, Bavaria, Germany
}

\begin{abstract}
A new LGS configuration termed Projected Pupil Plane Pattern $\left(P^{4}\right)$, together with the corresponding wavefront sensing and reconstruction method, is presented. A theoretical analysis and Monto Carlo simulation show the feasibility of this novel method for a 4m-class telescope and great promise for the next generation of ELTs. Also we carry out a simple laboratory test with only defocus aberration and compare the reconstructed phase with the corresponding result from a Shack-Hartmann WFS. A further high-order experiment will follow, as well as plans for an on-sky test at William Herschel Telescope (WHT), La Palma.
\end{abstract}

Keywords: Adaptive Optics, ELTs, LGS, $P^{4}$

\section{INTRODUCTION}

As the size of telescopes increases, the correction for the effects of atmospheric turbulence using Adaptive Optics (AO) become more and more critical. Laser Guide Stars (LGS) are a particularly important topic of research in $\mathrm{AO}$ systems as a means of sensing the distortion of an optical beam traveling in the Earth atmosphere without the need for a bright natural reference source. Conventionally, the laser is used to project a bright spot in the atmosphere and the backscattered wave-front is analyzed with a wave-front sensor (WFS) such as ShackHartmann WFS as though it came from a point source. A main difficulty with this LGS technique is that for high altitude turbulence layers, the patch of turbulence observed by the LGS will be different to that observed by the astronomical science target due to the finite LGS altitude. ${ }^{1}$ Thus the light traveling back to the telescope is through a cone instead of a column. This so-called cone effect becomes more pronounced for larger telescope diameters, such as the proposed next generation optical ground-based Extremely Large Telescopes (ELTs) with primary mirror diameters of over $30 \mathrm{~m}$.

Laser tomography AO (LTAO) then has been developed to mitigate the LGS cone effect, where several LGS have been generated simultaneously at different angular positions in the sky. Each LGS is associated to a dedicated WFS, and measurements from all wavefront sensors have been combined to estimate the 3D-mapped turbulence. The complexity and expense scales with the size of the telescope primary mirror. Also the LTAO requires a model for the vertical structure of $C_{n}^{2}$, and this model must be updated to keep up with the changes in the vertical structure of the atmosphere. At optical wavelengths the rate at which the model must be updated may become impracticable on some observatory sites.

Here we adopt a new LGS configuration and wavefront sensing method proposed by D. Buscher, G. Love and R. Myers called Projected Pupil Plane Patterns $\left(P^{4}\right)^{2}$ to directly measure wavefront distortions without requiring a model for the vertical structure. The key features of $P^{4}$ are that a parallel (or nearly parallel) laser beam is projected from the full primary aperture and that sensing takes place on the upward path. The methods therefore rely on an observable modulation of the scattered intensity by turbulence-induced phase distortions during upward propagation of the laser beam. Compared to LTAO $P^{4}$ is much less complex and cheaper and demonstrates progress towards a future optical-wavelength AO capability for ELTs.

A laboratory experiment of $P^{4}$ is ongoing now and an on-sky experiment will be carried out on the $4.2 \mathrm{~m}$ William Herschel Telescope (WHT), La Palma, in late 2018 or early 2019, and we will examine the use of Selex avalanche photo diodes with infrared laser light, which leaves then open the visible window for the actual astronomical 


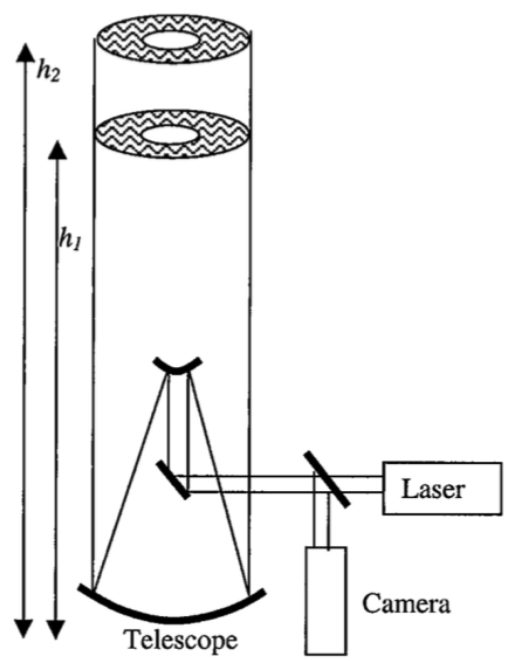

Figure 1. Schematic diagram of $P^{4}$ taken from D. F. Buscher et al. ${ }^{2}$ A laser beam is expanded to fill the pupil of the telescope and propagated through the telescope optics as a parallel beam upward through the atmosphere. When the laser pulse reaches an altitude of $h_{1}$ a snapshot of the Rayleigh backscattered radiation is taken with a camera focused at $h_{1}$, which will show a disk of illumination corresponding to the telescope pupil. When the pulse reaches an altitude of $h_{2}$, a second snapshot is taken with a camera focused at $h_{2}$ (better to use the same camera with optical modulation between the two planes).

observations.

In this paper we demonstrate the feasibility of $P^{4}$ method for a WHT $4 \mathrm{~m}$ telescope and investigate the performance under different conditions.

\section{2. $P^{4}$ THEORY}

The basic setup for $P^{4}$ is illustrated in Fig. 1, where a collimated beam is projected upwards from a telescope primary mirror into the atmosphere. As the parallel beam propagates, the intensity variations will develop according to the transport-of-intensity equation (TIE),

$$
k \partial_{z} I=-\nabla \cdot(I \nabla \phi),
$$

where $\nabla=\left(\partial_{x}, \partial_{y}\right), I$ is the intensity, $k=2 \pi / \lambda$ is the wavenumber and $\phi$ is the turbulence-introduced phase. On the basis of the TIE, if we know the distribution of intensity (i.e. $I$ ) and its $z$ derivative, the phase $\phi$ then can be retrieved. Here we adopt a linear reconstruction method proposed by T. Gureyev and K. Nugent. ${ }^{3}$

The simulation of $P^{4}$ method can be divided into three steps: firstly to propagate a collimated beam upwards to several different altitudes (a minimum of two is required) -termed upward propagation; then to reimage the Rayleigh backscattered intensity patterns at those altitudes through the same telescope by cameras conjugate at the corresponding heights -termed return path; finally to retrieve the distorted phase using the subtraction of the images from the cameras, which has been scaled to the same flux amount to guarantee the conservation of energy, as the intensity $z$ derivative in TIE -termed reconstruction. The major difference of $P^{4}$ and conventional LGS wave-front sensing, which is the combination of a focused LGS and Shack-Hartmann WFS, lies in the fact that the required signal for $P^{4}$ is generated by the upward propagation of the collimated laser beam, while the return path can be treated simply as a re-image process, i.e. a convolution of the atmospheric PSF and the intensity pattern on sky, and may affect the light backscattered patterns depending on the strength of the turbulence. However for the conventional LGS Shack-Hartmann wave-front sensing, the return path deals with the needed slope measurement while the upward propagation of the focused laser beam may introduce undesired 
LGS distortion.

Another unique phenomenon for $P^{4}$ is that the telescope primary mirror, which has been used to launch the laser beam, also is used to collect the scattered light from sky, as well as the light from the scientific object. Thus if we use a short-wavelength laser, say 589nm (typical for Sodium LGS), and infrared scientific camera, the fluorescence from the telescope optics then will cause interference for the science instrument. Therefore we have to play it another way round, using a pulsed laser longer than $800 \mathrm{~nm}$ such as Nd:YAG at $1064 \mathrm{~nm}$ and limit the science observations to shorter $\lambda$.

\section{3. $P^{4}$ SIMULATION RESULTS}

$P^{4}$ simulation modelling includes three parts: 1) upward propagation of a collimated beam to several altitudes (two here) through the atmospheric turbulence; ${ }^{4} 2$ ) the light is back-scattered (not collimated anymore) from these two layers, affected by the turbulence and imaged by a conjugate camera; 3 ) reconstruction process.

Regarding the return path, two issues should be taken into consideration: 1) the amount of flux scattered back (related to SNR), which can be calculated by LIDAR equation ${ }^{1}$ given the laser power, telescope diameter etc.; 2) the downward turbulence-introduced PSF, which can be obtained by the scaled Fourier transform of the generalized pupil $P(x, y)=I_{0}^{1 / 2} \exp (j \phi)$ according to ${ }^{5}$

$$
\operatorname{PSF}=\left|\mathcal{F}\left[P\left(\lambda h f_{x}, \lambda h f_{y}\right)\right]\right|^{2} .
$$

The averaged PSF with regards to different $r_{0}$ has been shown in Fig. 2. For $r_{0}=0.15 \mathrm{~m}$ at $550 \mathrm{~nm}$, the PSF width is approximately $0.04 \times 2=0.08 \mathrm{~m}$ and the required pixel number $N$ should be at most $D / 0.08=50$ for a $4 \mathrm{~m}$ telescope if the PSF is one pixel or less, thus has no influence on the $P^{4}$ signal. For $r_{0}=0.1 \mathrm{~m}$, and $0.2 \mathrm{~m}$, the required $N$ is 66 and 40 respectively.

During the return path the laser speckle pattern, produced by the diffuse reflections of laser light acting on materials such as paper, rough surfaces, or in media with a large number of scattering particles in space (for example the atmosphere), can be ignored since the time scale of atmospheric molecules moving is much smaller than the time scale of the turbulence changing, thus the laser speckle has been averaged out during the atmospheric integration time ( $\sim$ several ms). As for the reconstruction process, the calculation of the interaction matrix, can be theoretically obtained given $I_{0}$ according to Gureyev et al. ${ }^{3}$

For a conventional focused LGS combined with a Shack-Hartmann, a natural star is still required for tip-tilt correction, since a movement of LGS caused by the uplink propagation is observed by the Shack-Hartmann WFS as a tip-tilt aberration present in the atmosphere thus introducing wrong tip-tilt information. $P^{4}$ experiences similar problem, where the tip-tilt signal generated from the upward propagation (which is a movement of the intensity pattern) will be affected by the return path, therefore it is also necessary to use an extra natural star to provide tip-tilt information. Due to this reason from now on we only consider atmospheric aberrations without tip-tilt.

When introducing photon and read noise, a denoised process is demanded. Here a Guassian filter in the spatialfrequency domain has been used. Specifically we perform the Fourier transform to the intensity pattern on ground $I_{1}$ and $I_{2}$ separately, then multiplying a Guassian function $\left(\exp \left[-f^{2} / 2 s^{2}\right]\right.$ with the standard deviation $s=3.5$ ) before inverse Fourier transform, which leads to the denoised signal. The reconstructed phase before and after this denoise method is shown in Fig. 3 and we can see that the reconstructed phase from the denoised signal improves a lot.

We have integrated the $P^{4}$ model into soapy, which is a Monte Carlo Adaptive Optics simulation written in the Python programming language. ${ }^{6}$ A GUI of Soapy including $P^{4}$ model is shown in Fig. 4 with the parameters listed in Table 1. The averaged wavefront error from 100 random phase screens is $92 \mathrm{~nm}$ for $P^{4}$ and $112 \mathrm{~nm}$ for Shack-Hartmann WFS without noise. The corresponding result with photon and $3 e^{-}$read noise is $161 \mathrm{~nm}$ for $P^{4}$ and $131 \mathrm{~nm}$ for Shack-Hartmann WFS. 


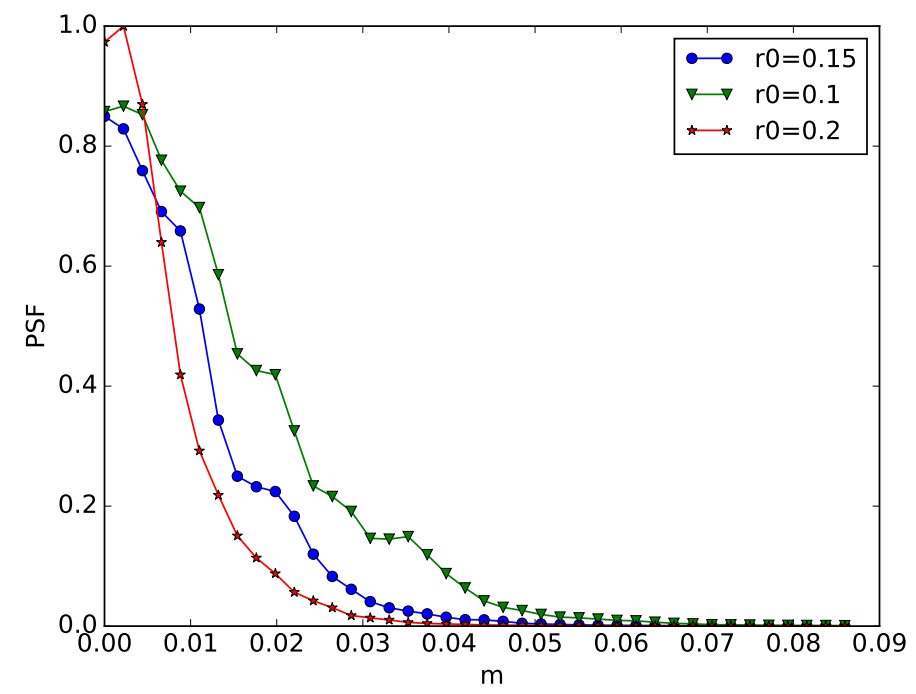

Figure 2. Return-path turbulence-introduced averaged PSF from 100 random phase screens.
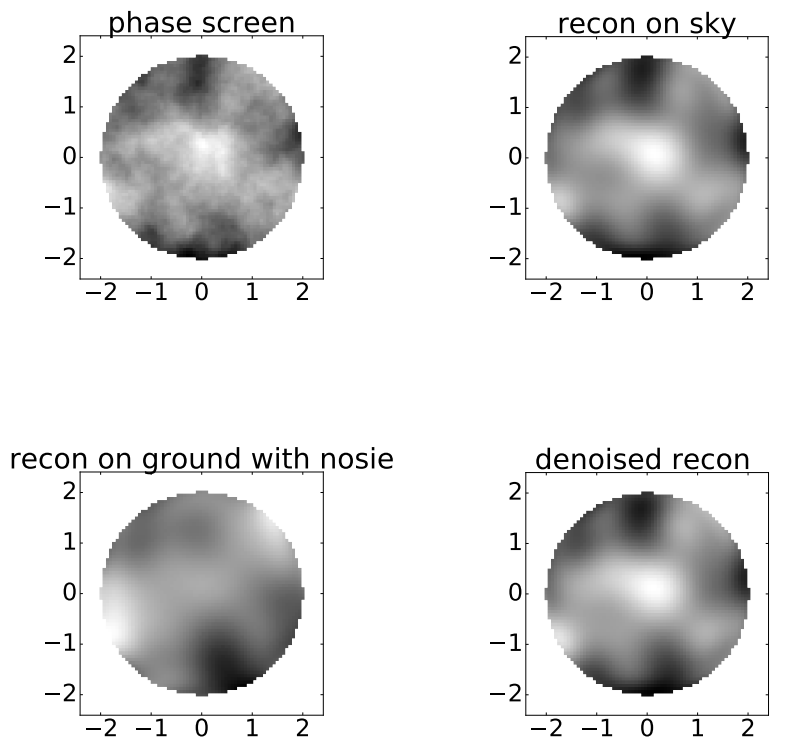

Figure 3. An example of $P^{4}$ reconstructed phase. The top-left is the original turbulence phase; the top-right is the reconstructed phase from the signal without noise; the bottom-left is the reconstructed phase from the signal with noise and the bottom-right is the reconstructed phase from the denoised signal. 
Table 1. Parameters for Soapy simulation platform. $P^{4}$ and Shack-Hartmann share the same parameters for telescope, atmosphere, DM and science camera.

\begin{tabular}{ll}
\hline Telescope & Atmosphere \\
\hline $\mathrm{D}=4 \mathrm{~m}$ & Phase screens height: $[0,5,10,15] \mathrm{km}$ \\
central obscuration: $1 \mathrm{~m}$ & Phase screens relative strength: $[0.5,0.3,0.2,0.1]$ \\
$128 \times 128$ pixels over pupil & $r_{0}=0.15 \mathrm{~m}$ \\
\hline $\mathrm{DM}$ & Science camera \\
\hline Zernike DM & $\lambda=0.8 \mathrm{um}$ \\
79 Zernike modes & $64 \times 64$ pixels \\
open loop & FOV: $2 "$ \\
& exposure time $2.5 \mathrm{~ms}$ \\
\hline$P^{4}$ & Shack-Hartmann WFS \\
\hline $32 \times 32$ pixels & LGS height 90km \\
$\lambda=1.06 \mathrm{um}$ & $\lambda=1.06$ um \\
$h_{1}=10 \mathrm{~km}$ & Subap Number $14 \times 14$ \\
$h_{2}=20 \mathrm{~km}$ & Pixel number per subap: 14 \\
laser pulse length $1 \mathrm{~km}$ & FOV per subap $5 "$ \\
pulsed laser power $20 \mathrm{~mJ}(20 \mathrm{~W})$ & \\
\hline
\end{tabular}

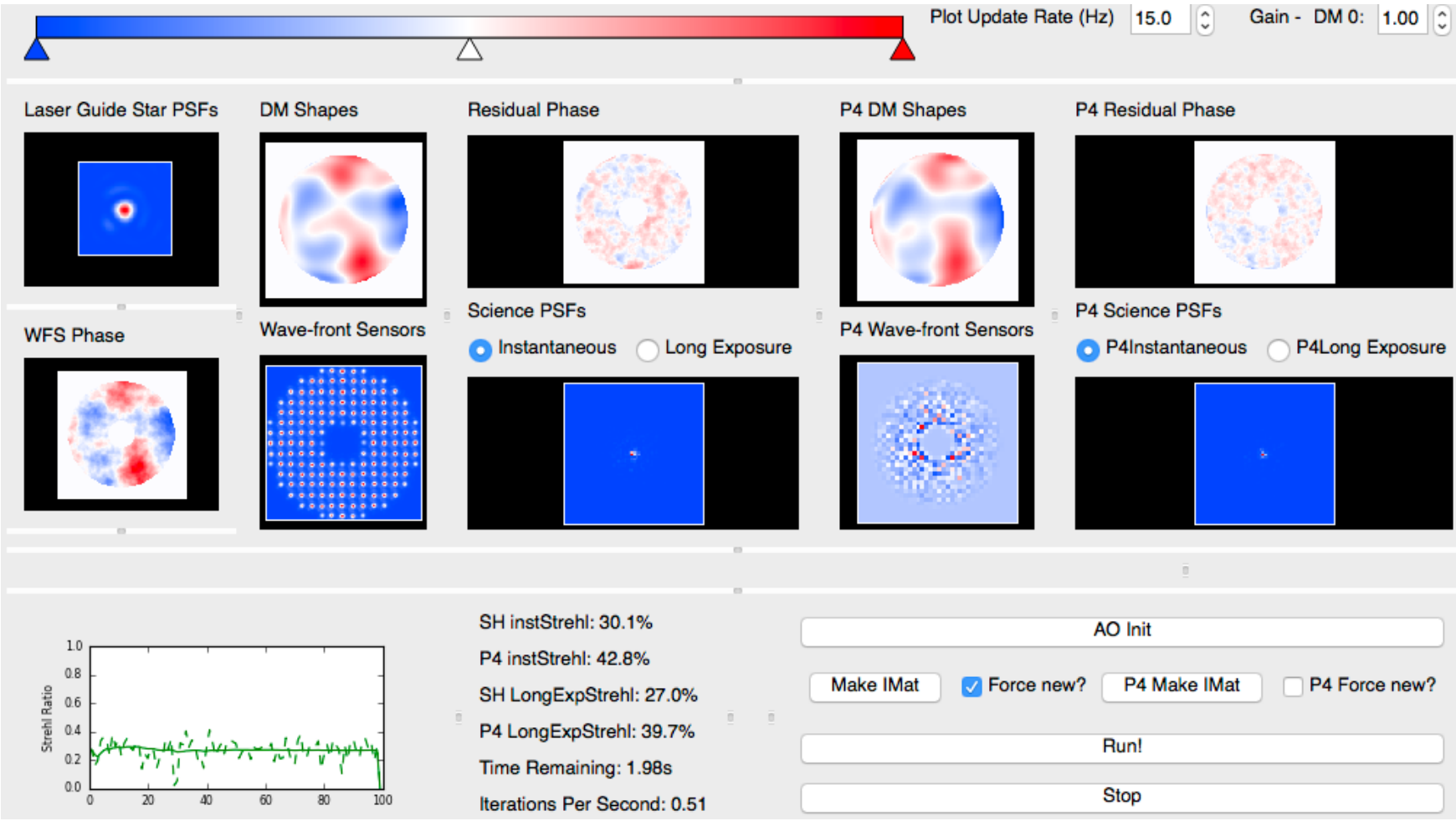

Figure 4. Soapy GUI including $P^{4}$ model. 


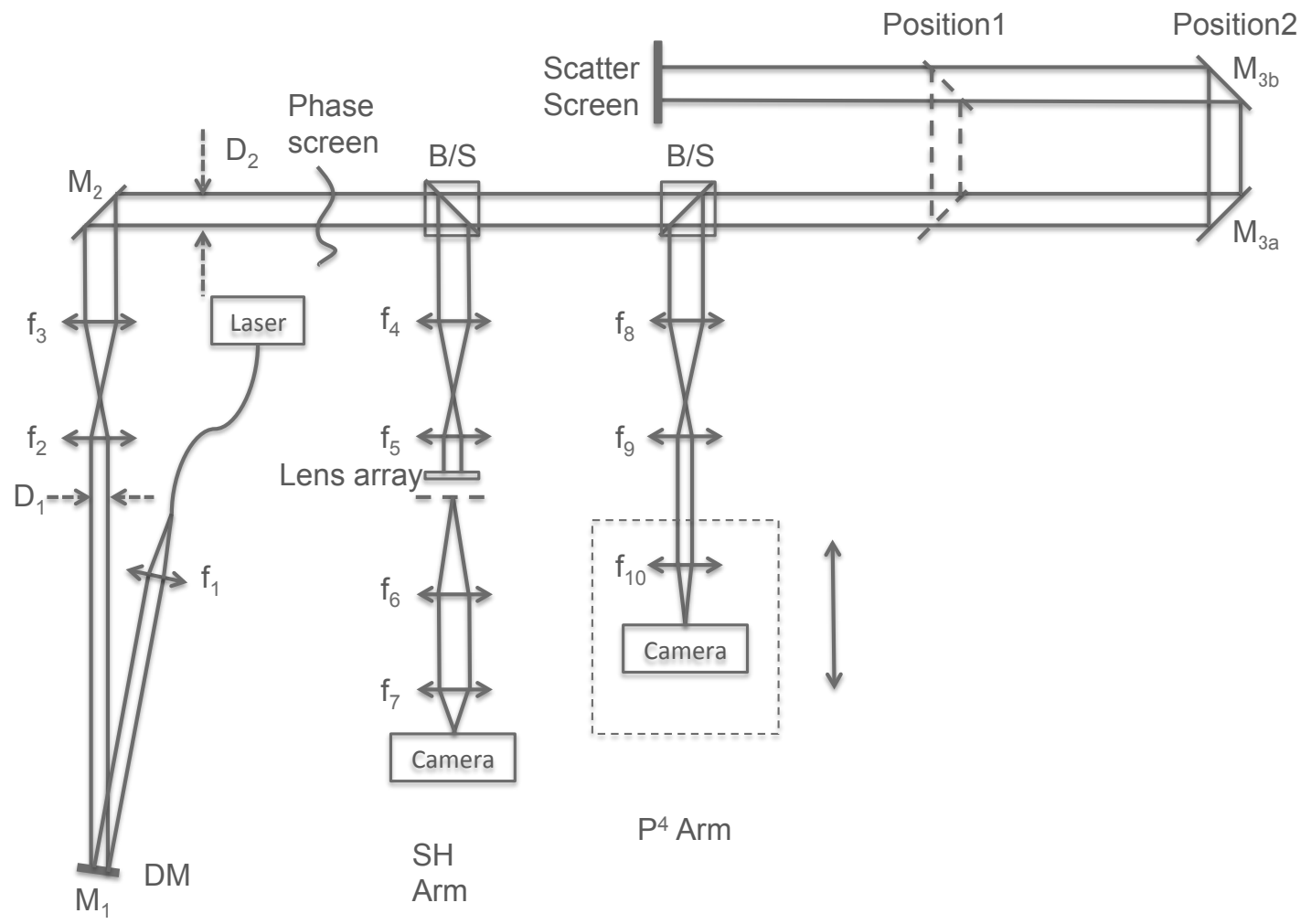

Figure 5. Reconstructed phase from defocus aberration.

\section{4. $P^{4}$ LABORATORY FEASIBILITY STUDY}

A preliminary laboratory experiment is ongoing now to verify the $P^{4}$ concept and the optical design is shown in Fig. 5. For now the DM has not been included but a Shack-Hartmann WFS has been used as a comparison to $P^{4}$. The pairs of $f_{2} \& f_{3}, f_{4} \& f_{5}, f_{6} \& f_{7}, f_{8} \& f_{9}$ are all used as pupil to pupil relay. For the $P^{4}$ path, the laser light goes though the second beam splitter $(\mathrm{B} / \mathrm{S})$ and propagates to the scatter screen, which is a reflective high material. The propagation distance can be controlled by moving $M_{3 a} \& M_{3 b}$ together. Then the scattered light will travel back by $M_{3 a} \& M_{3 b}$ and goes through the second $\mathrm{B} / \mathrm{S}$ into the $P^{4}$ path, where the intensity pattern on the scatter screen can be reimaged. $f_{8} \& f_{9}$ is to change the pupil size and $f_{10}$ is an imaging lens. By moving $M_{3 a} \& M_{3 b}$ from position 1 to position 2, the position and size of the image also changes. In this design we can simply move $f_{10}$ and the camera afterwards together on a rail to get the focused image of the intensity pattern without changing the image size on the camera. A simple test has been done using just defocus aberration and the result is shown in Fig. 6, which verifies the accuracy of $P^{4}$ method for this simple aberration.

\section{CONCLUSION}

We have demonstrated the feasibility of a new LGS configuration and wavefront sensing method, named Projected Pupil Plane Pattern $\left(P^{4}\right)$ by theoretical analysis, Monte Carlo simulation and a simple laboratory experiment. This new LGS wavefront sensing method shows a a great promise for the Extremely Large Telescopes due to its simplicity and speed. A further high-order experiment will be implemented in the lab, as well as an on-sky test on WHT.

\section{ACKNOWLEDGMENTS}

This work is funded by the Science and Technology Facilities Council (STFC). Many thanks to Yonghui Liang, NUDT, China. 

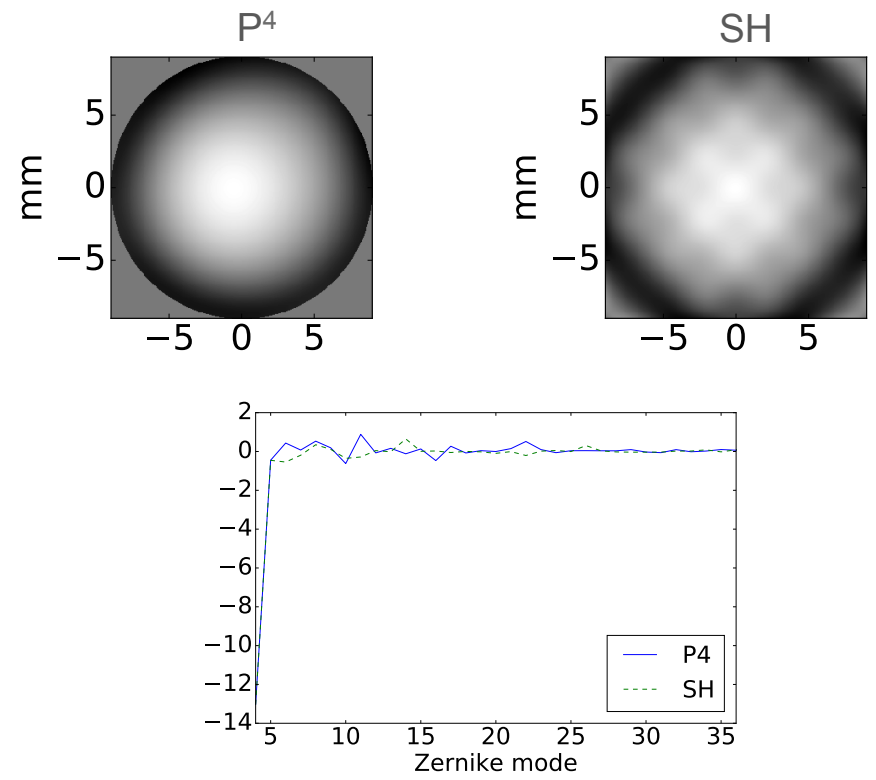

Figure 6. A schematic diagram of the laboratory experiment.

\section{REFERENCES}

[1] Hardy, J., [Adaptive Optics for Astronomical Telescopes], Oxford University Press, Oxford (1988).

[2] Buscher, D. F., Love, G. D., and Myers, R. M., "Laser beacon wave-front sensing without focal anisoplanatism," Optics Letters 27, 149-151 (2002).

[3] Gureyev, T. E. and Nugent, K. A., "Phase retrieval with the transport-of-intensity equation. ii. orthogonal series solution for nonuniform illumination," J. Opt. Soc. AM. A 13, 1670 (1996).

[4] Schmidt, J. D., [Numerical Simulation of Optical Wave Propagation], SPIE Press (2010).

[5] Roggermann, M. C., [Imaging through turbulence], CRC Press (1996).

[6] Reeves, A. P., "Laser guide star only adaptive optics," (2015). 